DOI: https://doi.org/10.26694/ics hu-ufpi.v3i1.11208

\title{
RECOMENDAÇÕES DE BIOSSEGURANÇA PARA A ATUAÇÃO DO CIRURGIÃO BUCOMAXILOFACIAL NO PERÍODO DE PANDEMIA DO CORONAVÍRUS
}

\author{
BIOSAFETY RECOMMENDATIONS FOR THE PERFORMANCE OF THE MAXILOFACIAL SURGEON DURING \\ THE CORONAVIRUS PANDEMIC PERIOD
}

Sérgio Éberson da Silva Maia ${ }^{1}$, Luís Paulo da Silva Dias², Patrick Saboia Beserra ${ }^{3}$, Laís Inês Silva Cardoso ${ }^{4}$, Kim $^{2}$ Rafael Veloso da Silva ${ }^{5}$, Thais Cristina Araújo Moreira ${ }^{6}$

${ }^{1}$ Cirurgião-Dentista pela UNILEÃO, Juazeiro do Norte- CE. Residente do Programa de Cirurgia e Traumatologia Bucomaxilofacial da Universidade Federal do Piauí. E-mail: sergioeberson@gmail.com ORCID iD: https://orcid.org/0000-0002-6609-1078

${ }^{2}$ Cirurgião Dentista pela UESPI de Parnaíba - PI. Residente do Programa de Cirurgia e Traumatologia Bucomaxilofacial da Universidade Federal do Piauí. E-mail: Ip.uespi@gmail.com

${ }^{3}$ Cirurgião Dentista pela UFPI, Teresina - PI. Residente do Programa de Cirurgia e Traumatologia Bucomaxilofacial da Universidade Federal do Piauí E-mail: patricksaboia@msn.com

${ }^{4}$ Cirurgiã Dentista pela UFMA, Universidade Federal do Maranhão, São Luís, MA. Residente do Programa de Cirurgia e Traumatologia Bucomaxilofacial da Universidade Federal do Piauí. E-mail: laisinescardoso@gmail.com

${ }^{5}$ Cirurgião Bucomaxilofacial do Hospital Regional Justino Luz, Picos - PI. E-mail: kimveloso@hotmail.com

${ }^{6}$ Cirurgiã Bucomaxilofacial do Hospital Universitário da UFPI, Teresina - PI. E-mail: thaiscam@yahoo.com

\section{RESUMO}

As principais formas de transmissão do Coronavírus - COVID-19 se dão pelo contato com aerossóis gerados pelas vias respiratórias superiores e cavidade oral de pessoas contaminadas, condição que coloca o Cirurgião Bucomaxilofacial como um dos profissionais mais expostos a contaminação. $O$ atendimento das afecções maxilofaciais traumáticas e não traumáticas demandam pela adoção de novos protocolos de biossegurança frente ao manejo desses casos. O objetivo deste estudo é elaborar um protocolo de biossegurança voltado para a prática da Cirurgia e Traumatologia Buco-Maxilo-facial no contexto da pandemia do COVID-19, por meio de uma revisão integrativa da literatura. Metodologia: O presente trabalho trata-se de um estudo transversal descritivo sob a forma de revisão integrativa da literatura, para compor a amostra foram selecionados artigos com tema voltado a atuação do cirurgião Bucomaxilofacial no contexto da pandemia do covid-19. As bases de pesquisa foram a SCIELO Scientific Electronic Library Online - SCIELO e Public medline - PUBMED, nos idiomas português e inglês. Conclusão: $O$ uso de equipamentos de proteção individual juntamente com um manejo seguro em todas as fases do atendimento, bem como, a estratificação do atendimento de acordo com a severidade dos casos são estratégias mais viáveis neste momento, no âmbito intra-hospitalar. Medidas de saúde coletiva, tais como, o isolamento social, uso de máscaras, o maior rigor com higiene pessoal, sobretudo, das mãos e do contato direto entre os indivíduos juntamente com o início de estudos para o desenvolvimento de vacinas também são de suma importância para diminuir a incidências dos casos.

DESCRITORES: Coronavírus. Cirurgia Maxilofacial. Pandemia. 


\section{ABSTRACT}

The main forms of transmission of the Coronavirus - COVID-19 occur through contact with aerosols generated by the upper respiratory tract and the oral cavity of contaminated people, a condition that places of the maxilofacial Surgeon as one of the professionals most exposed to contamination. The treatment of traumatic and non-traumatic maxillofacial disorders requires the adoption of new biosafety protocols in relation to management in these cases. The objective of this study is to develop a biosafety protocol aimed at the practice of the Traumatology and Maxilofacial Surgery in the context of the COVID-19 pandemic, through an integrative literature review. Methodology: The present work is a cross-sectional descriptive study in the form of an integrative literature review. To compose the sample, articles were selected with a theme aimed at the performance of the maxilofacial surgeon in the context of the covid-19 pandemic. The research bases were SCIELO Scientific Eletronic Library Online - SCIELO and Public medline - PUBMED, in Portuguese and English. Conclusion: The use of personal protective equipment together with safe handling in all phases of care, as well as the stratification of care according to the severity of the cases are the most viable clinic at this moment, within the hospital. Collective health measures, such as social isolation, the use of masks, greater strictness with personal hygiene, especially hands and direct contact between those integrated together with the initiation of studies for the development of vaccines, are also of paramount importance. to decrease the incidence of cases.

KEYWORDS: Coronavirus. Maxilofacial surgery. Pandemic.

\section{Como citar este artigo (Vancouver):}

Maia SES, Dias LPS, Beserra PS, Cardoso LIS, Silva KRV, Moreira TCA. Recomendações de biossegurança para a atuação do cirurgião bucomaxilofacial no período de pandemia do Coronavírus. J. Ciênc. Saúde [internet]. 2020 [acesso em: dia mês abreviado ano]; JCS HU-UFPI. Ed. Espec. 2020; 3(Supl.2):e-11208. Disponível em: DOI: https://doi.org/10.26694/jcs hu-ufpi.v3i1.11208

\section{(cc) BY}


hospitalares que atuam no manejo e tratamento de afeç̧ões do sistema estomatognático, essas compreendidas pelas patologias neoplásicas, císticas, infecciosas, trauma oral e maxilofacial bem como outras Os primeiros casos de coronavírus surgiram na China, na cidade de Wuhan no final do ano de 2019, foi identificado como SARS-CoV-2, sendo um subtipo da linhagem B (Betacoronavírus), causa a síndrome respiratória aguda grave (SARS) que apresenta alto potencial de transmissibilidade entre os humanos, nesse contexto surgiram caso em todo o mundo, configurando-se como uma pandemia ${ }^{1}$.

A pandemia gerada pela infecção pelo COVID-19 é um problema de saúde pública de grande significância para todas as nações, em virtude da incidência crescente, as medidas de controle adotadas e recomendadas pela OMS e demais órgãos da saúde são de suma importância para a minimização dos agravos e determinam a inclusão e atuação de todas as categorias da saúde ${ }^{2}$.

O primeiro caso registrado no Brasil foi datado em 25 de fevereiro de 2020, na cidade de São Paulo em um paciente de 61 anos que havia chegado ao país após uma viagem à Itália, sendo diagnosticado a partir de exames laboratoriais. Hoje no Brasil de acordo com o Ministério da Saúde (MS) foram registrados 2.394.513 casos, com 86.449 óbitos, tendo uma incidência de 1139,4 a cada 100.000 habitantes e uma taxa de mortalidade de 41,1 a cada 100.000 habitantes. 0 estado do Piauí apresenta 45.389 casos, com 1228 óbitos até o momento ${ }^{3}$.

Tendo em vista que as principais formas de transmissão do COVID-19 se dão pelo contato com aerossóis gerados pelas vias respiratórias superiores e cavidade oral de pessoas, relação que coloca o Cirurgião Bucomaxilofacial como um dos profissionais mais expostos a contaminação. $O$ atendimento do trauma facial é o principal agravo conduzido pela especialidade, outras afecções também são privativas da assistência, tais como infecções odontogênicas, essas condições clinicas demandam de atendimento de urgência e/ou emergência ${ }^{4}$.

A Cirurgia e Traumatologia Buco-Maxilo-Facial é uma especialidade reconhecida da Odontologia e compõe o quadro de especialidades ambulatoriais e

Nesse contexto, a atuação profissional é de suma importância nas unidades hospitalares, entretanto, em uma situação de calamidade pública pela pandemia instalada do COVID 19 é plausível considerar que os pacientes vítimas de acidentes e outras circunstâncias que provoquem traumatismos na face são passiveis de contaminação pelo vírus na comunidade e/ou no ambiente hospitalar e no manejo destes pacientes pode ocorrer contaminação dos profissionais ${ }^{6,7}$.

O objetivo deste estudo é elaborar um protocolo de biossegurança voltado para a pratica da Cirurgia e Traumatologia Buco-Maxilo-facial com base nas recomendações das entidades representantes da especialidade e também da literatura relacionada a procedimentos cirúrgicos e atuação do cirurgião Bucomaxilofacial no contexto da pandemia do COVID19, por meio de uma revisão integrativa da literatura.

\section{METODOLOGIA}

O presente trabalho trata-se de um estudo transversal descritivo sob a forma de revisão integrativa da literatura, para compor a amostra foram selecionados artigos com tema voltado a atuação do cirurgião Bucomaxilofacial no contexto da pandemia do covid-19. As bases de pesquisa foram a SCIELO Scientific Electronic Library Online - SCIELO e Public medline PUBMED, com o uso dos descritores: Coronavírus; COVID-19; Cirurgia Bucomaxilofacial e Pandemia bem como os respectivos termos em inglês: Coronavirus; COVID-19; maxilofacial surgery; pandemic. Amostra foi composta de acordo com os critérios de inclusão e exclusão de acordo com o Quadro 01.

QUADRO 1 - Critérios de inclusão e exclusão

\begin{tabular}{lll}
\hline \multicolumn{2}{c}{ Critérios de Inclusão } & \multicolumn{2}{c}{ Critérios de exclusão } \\
\hline Artigos completos de & $\begin{array}{l}\text { Comentários } \\
\text { livre acesso. }\end{array}$ & $\begin{array}{l}\text { especialistas, relatos de } \\
\text { caso. }\end{array}$ \\
\end{tabular}




\begin{tabular}{|c|c|}
\hline Artigos publicados a & Artigos que não abordam \\
\hline $\begin{array}{l}\text { partir pandemia do } \\
\text { COVID-19. }\end{array}$ & $\begin{array}{l}\text { protocolos e ou } \\
\text { orientações para cirurgia. }\end{array}$ \\
\hline $\begin{array}{ll}\text { Artigos que tratem de } & \text { de } \\
\text { condutas } & \text { biossegurança }\end{array}$ & $\begin{array}{l}\text { Artigos que não tratam de } \\
\text { medidas coletivas de } \\
\text { biossegurança. }\end{array}$ \\
\hline $\begin{array}{l}\text { relacionados } \\
\text { procedimentos }\end{array}$ & \\
\hline cirúrgicos pela CTBMF. & \\
\hline
\end{tabular}

Fonte: Autoria própria.

Para compor as referências foram priorizados artigos que trazem as orientações de entidades nacionais e internacionais que representam a especialidade e ou recomendações adotadas em serviços especializados em Cirurgia e Traumatologia Buco-Maxilo-Faciais. Os dados epidemiológicos sobre os casos de covid-19 no brasil e no estado do Piauí foram coletados do site do Ministério da Saúde (MS)

\section{RESULTADOS}

Com base na análise dos estudos da revisão bibliográfica estão descritas as principais recomendações e orientações para a atuação do Cirurgião Bucomaxilofacial, no que diz respeito a biossegurança na prática cirúrgica e clínica no âmbito hospitalar frente a pandemia do coronavírus (COVID19). Na tabela 01 estão descritas a principais recomendações.

TABELA 1 - Recomendações da atuação do cirurgião Bucomaxilofacial no atendimento durante a pandemia do COVIS-19.

(Continua)

\begin{tabular}{|c|c|}
\hline ESTUDO/ARTIGO & RECOMENDAÇÕES/ORIENTAÇÕES \\
\hline $\begin{array}{l}\text { CHIGURUPATI et al., } \\
2020 .\end{array}$ & $\begin{array}{l}\text { - Triagem e testagem dos pacientes antes da cirurgia. } \\
\text { - Treinamento de residentes cirurgiões no manejo do COVID-19. } \\
\text { - Uso de EPI completo no atendimento (máscara cirúrgica, PFF2/N-95, } \\
\text { face-shield, óculos de proteção, capote descartável). } \\
\text { - Realizar somente cirurgias de urgência e emergência. }\end{array}$ \\
\hline HOLMES et al., 2020. & $\begin{array}{l}\text { - Suspender atendimentos eletivos. } \\
\text { - Usar fixação reabsorvível nas fraturas dos ossos da face. } \\
\text { - Evitar acessos extensos. } \\
\text { - Evitar acessos intra-orais. } \\
\text { - } \quad \text { Reduzir número de cirurgiões por cirurgia. }\end{array}$ \\
\hline EBBEN et al., 2020 & $\begin{array}{l}\text { - Suspender procedimentos eletivos. } \\
\text { - Manter atendimento de urgência emergência. } \\
\text { - Reduzir número de cirurgiões em cirurgias. } \\
\text { - Manter equipes com membros fixos. } \\
\text { - Afastar os profissionais com sintomas respiratórios. }\end{array}$ \\
\hline $\begin{array}{l}\text { BLACKHALL et al., } \\
2020\end{array}$ & $\begin{array}{l}\text { - Redução da carga-horaria de urgência e emergência dos cirurgiões } \\
\text { Bucomaxilofaciais. } \\
\text { - Suspensão de procedimentos eletivos. } \\
\text { - Parte da assistência feita por tele atendimento. }\end{array}$ \\
\hline
\end{tabular}


TABELA 1 - Recomendações da atuação do cirurgião Bucomaxilofacial no atendimento durante a pandemia do COVIS-19.

ESTUDO/ARTIGO

\begin{tabular}{|c|c|}
\hline WANG et al., 2020. & $\begin{array}{l}\text { - Reduzir número de membros das equipes cirúrgicas. } \\
\text { - Antissepsia com lodopovidona tópico (PVPI). } \\
\text { - Evitar acessos a cavidade nasal e oral. } \\
\text { - Dar preferência tratamentos conservadores quando possível. } \\
\text { - Evitar osteotomia e uso de cautério. } \\
\text { - Fazer antissepsia no trans-cirúrgico quando houver manipulação de } \\
\text { mucosa. }\end{array}$ \\
\hline ALLEVI et al., 2020 & $\begin{array}{l}\text { - Manutenção de cirurgias de emergência. } \\
\text { - Manutenção de cirurgias oncológicas, tumores benignos e malignos. } \\
\text { - Suspender cirurgias eletivas. } \\
\text { - Ordenar demanda cirúrgica por gravidade dos casos. }\end{array}$ \\
\hline EDWARDS et al.,2020 & $\begin{array}{l}\text { - Dar preferência a tratamentos conservadores no trauma de face. } \\
\text { - Uso de EPI completo (máscara cirúrgica, PFF2/N-95, face-shield, } \\
\text { óculos de proteção, capote descartável). } \\
\text { - Articular com outras equipes o atendimento de lesões comuns a área } \\
\text { de atuação dando preferência a intervenção já no atendimento de } \\
\text { urgência. }\end{array}$ \\
\hline XAVIER et al., 2020 & $\begin{array}{l}\text { - Triagem e testagem dos pacientes antes das cirurgias. } \\
\text { - Tricotomia da face nos homens. } \\
\text { - Redistribuição a funções entre a equipe para minimizar o tempo de } \\
\text { - } \quad \text { Realizarar cirurgias somente de urgência e emergência. }\end{array}$ \\
\hline CAREDDU et al., 2020 & $\begin{array}{l}\text { - Triagem e testagem dos pacientes antes da cirurgia. } \\
\text { - Realizar cirurgias somente de urgência e emergência. } \\
\text { - Uso de EPI completo (máscara cirúrgica, PFF2/N-95, face-shield, } \\
\text { óculos de proteção, capote descartável). }\end{array}$ \\
\hline PANESAR et al., 2020. & $\begin{array}{l}\text { - Triagem e testagem dos pacientes antes da cirurgia. } \\
\text { - Uso de EPI completo (máscara cirúrgica, PFF2/N-95, face-shield, } \\
\text { óculos de proteção, capote descartável). } \\
\text { - Treinamento de cirurgiões e residentes no manejo do covid-19. }\end{array}$ \\
\hline
\end{tabular}

Fonte: autoria própria. 
QUADRO 02- principais medidas de prevenção no manejo de pacientes atendidos pela CTBMF.

\section{Medidas de proteção no manejo de pacientes atendidos e operados pela CTBMF frente a pandemia do} COVID-19.

01 - Uso completo de EPI's (Máscara N95, N99, N100, PFF2 ou PFF3, gorro, avental descartável, óculos de proteção, luvas e ou protetor facial).

02 - Realizar exame clinico direcionado de maneira adequada, sem uso de jatos de ar ou água para minimizar a formação de aerossóis, orientar aos pacientes que colaborem evitando tossir e ou espirrar no momento do exame, após isso na necessidade de internação os mesmos devem permanecer de máscara.

03 - Quando necessária intervenção cirúrgica em pacientes com fraturas nos ossos da face, dar preferência a tratamentos conservadores e menos invasivos (BMM, odontossinteses e contenções).

04 - Pacientes hospitalizados devem ter sua permanência abreviada após a resolução da sua condição clínica. 05 - Aos pacientes que forem indicados tratamentos conservadores devem ser direcionados ou encaminhados aos serviços ambulatoriais de acompanhamento.

06 - Quando a realização de procedimento cirúrgico sob anestesia geral o profissional deve proceder a paramentação na seguinte sequencia: (lavagem das mãos, colocação de máscara N95, máscara cirúrgica por cima, óculos de proteção, face-shield, antissepsia cirúrgica das mãos, avental cirúrgico e luvas estéreis).

07 - A intubação é considerada um procedimento de geração de gotículas, o cirurgião dever entrar na sala 20 minutos após a intubação.

08 - Dar preferência a antissepsia oral e facial com PVPI a 10 \% na forma tópica.

09 - A cirurgia deve ser conduzida por um cirurgião experiente com a equipe mínima necessária, para evitar aglomeração desnecessária de profissionais.

10 - Na necessidade de acessos cirúrgicos, dar preferência a acessos extra-orais, evitando ao máximo a manipulação da cavidade oral e via aérea superior.

12 - Evitar a irrigação demasiada com soluções e aspiração da cavidade oral.

13 - Os pacientes sob assistência da CTBMF que apresentarem sintomas relacionados ao COVID-19, devem ser testados para o vírus e se confirmados o tratamento pela CTBMF caso não seja de extrema urgência deve ser postergado.

Recomendações baseadas nas orientações do Colégio Brasileiro de Cirurgia e Traumatologia Buco-maxilo-faciais (CBCTBMF), American Association of Oral and Maxillofacial Surgeons (AAOMS), French Society of Stomatology, MaxilloFacial Surgery and Oral Surgery (SFSCMFCO) e AO Foundation Craniomaxilfacial - AOCMF 


\section{DISCUSSÃO}

A Cirurgia e Traumatologia Buco-Maxilo-Facial (CTBMF) é uma especialidade que trata de patologias da região maxilofacial e seus anexos, tais como: traumatismos, deformidades dentofaciais (congênitas ou adquiridas), cistos e tumores dos maxilares, manifestações orais de doenças sistêmicas, síndromes que afetam a face a cavidade oral, alterações do crescimento facial, disfunções dores orofaciais dentre outras ${ }^{8,9}$.

No âmbito hospitalar o Cirurgião Bucomaxilofacial atua no atendimento de urgência e emergência exercendo sua formação como traumatologista da face, bem como, em cirurgias eletivas. A pandemia pelo novo Coronavírus modificou a atuação de diversas especialidades e centros hospitalares, fazendo com que novos protocolos sejam adotados no intuito de diminuir o risco de contaminação ${ }^{10,11}$.

Todos os pacientes atendidos pela CTBMF em âmbito hospitalar devem ser considerados como potencial contaminação pelo Coronavírus (SARS-CoV-2), dessa forma o profissional deve realizar o atendimento da maneira mais segura com uso completo de EPI's e minimizando manobras e condutas com maior risco de contaminação sobretudo da manipulação da cavidade oral e nasal ${ }^{12}$.

Yang et $\mathrm{al}^{1}$, categorizaram os pacientes sob assistência da CTBMF em quatro grupos distintos, sendo eles, 01Pacientes críticos: compreendem os casos de fraturas expostas, infecções agudas e neoplasias em condições de risco para obstrução das vias aéreas, para esse grupo a cirurgia deve ocorrer em caráter de emergência. 02Pacientes fraturas fechadas sem comprometimento de via aérea e ou hemorragia presente, na condição de sistemicamente estáveis. 03-Pacientes com diagnóstico de neoplasias malignas, osteomielite e outras infecções crônicas, necessitam de intervenção rápida. Os grupos 02 e 03 devem ser triados e testados para covid-19 caso apresentem sintomas, se exame positivo a cirurgia deve ser postergada e o paciente encaminhado a unidade de referência para tratamento do quadro clinico. 04-
Pacientes portadores de Deformidade dentofaciais, fissuras labiopalatais e tumores benignos, esses devem ser monitorados periodicamente e o procedimento cirúrgico realizado em caráter eletivo de acordo com remissão da pandemia e em melhores condições de segurança.

Observou-se que a maioria dos estudos aqui citados advogam pela suspensão dos procedimentos eletivos nesse período de pandemia. De acordo com Zhao e $\mathrm{Gao}^{13}$, procedimentos como cirurgias corretivas em pacientes com fissuras labiopalatais e deformidade dentofaciais (DDF) devam ser proteladas até um momento onde ocorra uma diminuição dos índices de contagio pelo SARS-CoV-2 e existam estratégias de prevenção mais efetivas.

Zimmermann et $\mathrm{al}^{14}$, relataram que os procedimentos cirúrgicos devem ser estratificados de acordo com a severidade, devendo ser prioridade cirurgias de emergência em pacientes com fraturas expostas não passiveis de redução fechada, infecções profundas da região de cabeça e pescoço, fraturas orbitarias como comprometimento da função ocular e tumores que causem obstrução das vias aéreas.

De acordo com Blackhall ${ }^{15}$ e colaboradores, evidenciouse que o número de atendimento de urgências traumáticas relacionadas a acidentes de transito e trabalho reduziram, no entanto, acidentes domésticos, agressão física e infecções aumentaram neste período de pandemia, isso corrobora pela manutenção dos atendimentos em prontos socorros pela CTBMF.

Todos os estudos apontam quanto a necessidade do uso de equipamentos de proteção individuais completos (EPI), tais como: máscara cirúrgica, PFF2/N-95, faceshield, óculos de proteção, capote descartável e capuz para cabeça e pescoço. De acordo com a Sociedade Francesa de Estomatologia e Cirurgia Bucomaxilofacial - SFSCMFCO ${ }^{16}$, o uso de mascaras do tipo PFF2, PFF3 ou N95, óculos de proteção e protetor facial são extremante necessários durante 0 atendimento, sobretudo em procedimentos cirúrgicos, em virtude dos procedimentos da cavidade oral gerarem gotículas e 
aerossóis que podem ficar dispersos no ambiente, favorecendo a contaminação dos profissionais.

A adoção dessas medidas é recomendada pela Organização Mundial de Saúde (OMS) e entidades representativas da Cirurgia e Traumatologia BucoMaxilo-facial, dentre elas: Colégio Brasileiro de Cirurgia e Traumatologia Buco-Maxilo-Faciais (CBCTBMF), American Association of Oral and Maxillofacial Surgeons (AAOMS), French Society of Stomatology, Maxillo-Facial Surgery and Oral Surgery (SFSCMFCO) e AO Foundation Craniomaxilfacial - AOCMF, bem como da experiência vivenciada em grandes centros em todo o mundo e elucidada através dos estudos citados nesse trabalho.

\section{CONCLUSÃO}

A Cirurgia e Traumatologia Buco-Maxilo-Facial é uma especialidade de suma importância na assistência hospitalar, entretanto, neste momento de pandemia pelo covid-19, novos protocolos devem ser adotados objetivando a manutenção do atendimento aos casos de urgência e emergência de maneira segura a profissionais e pacientes.

O uso de equipamentos de proteção individual juntamente com um manejo seguro em todas as fases do atendimento, bem como, a estratificação do atendimento de acordo coma severidade dos casos são estratégias mais viáveis neste momento, no âmbito intra-hospitalar. Medidas de saúde coletiva, tais como, o isolamento social, testes de diagnóstico, uso de máscaras, o maior rigor com higiene pessoal, sobretudo, das mãos e do contato direto entre os indivíduos juntamente com o início de estudos para o desenvolvimento de vacinas também são de suma importância para diminuir a incidência dos casos em todo mundo, sendo essas as principais recomendações adotadas pela OMS.

Todas as ações de saúde devem se voltadas para elaboração de medidas de controle e prevenção, nesse contexto a capacitação dos profissionais, distribuição de equipamentos de proteção juntamente com estabelecimento de métodos de diagnóstico são as medidas mais plausíveis

\section{REFERÊNCIAS}

1. Yang $Y$, Soh HY, Cai ZG, Peng $X$, Zhang $Y$, Guo $\mathrm{CB}$. Experience of Diagnosing and Managing Patients in Oral Maxillofacial Surgery during the Prevention and Control Period of the New Coronavirus Pneumonia. Chin J Dent Res. 2020;23(1):57-62.

2. World Health Organization. WHO. Novel Coronavirus (2019-nCoV) technical guidance, 2020. Disponível em: https://www.who.int/emergencies/diseases/novelcoro navirus-2019.

3. Ministério da Saúde (BR). Disponível em: https://covid.saude.gov.br/

4. Edwards SP, Kasten S, Nelson C, Elner V, McKean E. Maxillofacial Trauma Management During COVID-19: Multidisciplinary Recommendations. Facial Plast Surg Aesthet Med. 2020;22(3):157-159.

5. AO CMF International Task Force Recommendationson Best Practices for Maxillofacial Procedures during COVID-19 Pandemic. 26 March 2020. https://www.aofoundation.org/what-wedo/covid-19-resources-for

6. AAOMS MemberAlert: COVID-19 Guidance for OMS Practices. March 17, 2020. Disponível em: https://www.aaoms.org/news/member-alert-covid-19guidance-for-oms-practices

7. Colégio Brasileiro de Cirurgia e Traumatologia Buco-maxilo-Faciais -COVID-19 - Guia de Práticas em CTBMF, 30.03.2020. Disponível em: https://www.bucomaxilo.org.br/

8. Costa SM, Lacerda GT, Villafort RN, Silveira RL, Amaral MBF. We Know About COVID-19: Oral and Maxillofacial Surgeons Survey [published online ahead of print, 2020 May 28]. J Craniofac Surg. 2020;10.1097. 
9. Panesar K, Dodson T, Lynch J, Bryson-Cahn C, Chew L, Dillon J. Evolution of COVID-19 Guidelines for University of Washington Oral and Maxillofacial Surgery Patient Care. J Oral Maxillofac Surg. 2020;78(7):1136-1146.

10. Zeng L, Su T, Huang L. Strategic plan for management in oral and maxillofacial surgery during COVID-19 epidemic. Oral Oncol. 2020;105:104715.

11. Saggese NP, Cardo VA. A perspective from a NYC chief oral and maxillofacial surgery resident during the COVID-19 pandemic. Br J Oral Maxillofac Surg. 2020;58(6):730-731.

12. Patel NA, Ji YD, Odera SL. The Role of Oral and Maxillofacial Surgeons in COVID-19 Response. J Oral Maxillofac Surg. 2020;78(7):1052-1053.

13. Zhao Z, Gao D. Precaution of 2019 novel coronavirus infection in department of oral and maxillofacial surgery. Br J Oral Maxillofac Surg. 2020;58(3):250-253.

14. Zimmermann M, Nkenke E. Approaches to the management of patients in oral and maxillofacial surgery during COVID-19 pandemic. J Craniomaxillofac Surg. 2020;48(5):521-526.

15. French Society of Stomatology, Maxillo-Facial Surgery and Oral Surgery (SFSCMFCO). Practitioners specialized in oral health and coronavirus disease 2019: Professional guidelines from the French society of stomatology, maxillofacial surgery and oral surgery, to form a common front against the infectious risk. J Stomatol Oral Maxillofac Surg. 2020;121(2):155-158.

16. Blackhall KK, Downie IP, Ramchandani P, et al. Provision of Emergency Maxillofacial Service During the COVID-19 Pandemic: A Collaborative Five Centre UK Study. Br J Oral Maxillofac Surg. 2020;58(6):698703.

17. Allevi F, Dionisio A, Baciliero $U$, et al. Impact of COVID-19 epidemic on maxillofacial surgery in Italy. $\mathrm{Br}$ J Oral Maxillofac Surg. 2020;58(6):692-697.
18. Chigurupati R, Panchal N, Henry AM, et al. Considerations for Oral and Maxillofacial Surgeons in COVID-19 Era: Can We Sustain the Solutions to Keep Our Patients and Healthcare Personnel Safe? [published online ahead of print, 2020 May 24]. J Oral Maxillofac Surg. 2020;S0278-2391(20)30551-6.

19. Holmes S, Bhatti N, Bhandari R, Chatzopoulou $D$. Toward a consensus view in the management of acute facial injuries during the Covid-19 pandemic. $\mathrm{Br} \mathrm{J}$ Oral Maxillofac Surg. 2020;58(5):571-576. doi:10.1016/j.bjoms.2020.03.024

20. Ebben S, Hussain RA, Miloro M, Callahan N. The UIC COVID Coverage Protocol: A Technical Note for Pandemic Oral and Maxillofacial Surgery Call Coverage. J Oral Maxillofac Surg. 2020;78(7):1044-1046. doi:10.1016/j.joms.2020.04.004

21. Wang TT, Moon HS, Le A, Panchal N. Proceedings of the OMS COVID-19 Response Conference [published online ahead of print, 2020 May 11]. J Oral Maxillofac Surg. 2020;78(8):1268-1274.

22. Xavier TB, Barbosa GM, da Silva BBP, Daroz BG, dos Santos YP, Neto NC, Pontes HAR. Protocolo de Tratamento Odontológico na Cirurgia e Traumatologia Buco-Maxilo-Facial no Contexto do COVID-19/Dental Treatment Protocol in Buco-Maxillofacial Surgery in the Context of COVID-19. Braz. J. Health Review, 2020;3(3): 4484-4500.

23. Careddu R, Ciaschetti M, Creavin G, Molina F, Plotino G. COVID-19 and dental practice: overview and protocols during pandemic. G Ital Endodon [Internet]. 2020Apr.16 [cited 2020Jul.31];34(1)

Fontes de financiamento: Não

Conflito de interesse: Não

Aceito: 06/10/2020

Publicação: 23/10/2020

Endereço para correspondência: Sérgio Éberson da

Silva Maia. Juazeiro do Norte- CE. E-mail:

sergioeberson@gmail.com 